\title{
PTP1B: a new therapeutic target for Rett syndrome
}

\author{
Lutz Tautz
}

NCI-Designated Cancer Center, Sanford-Burnham Medical Research Institute, La Jolla, California, USA.

\begin{abstract}
Rett syndrome (RTT) is an X-linked neurodevelopmental disorder that is characterized by successive loss of acquired cognitive, social, and motor skills and development of autistic behavior. RTT affects approximately 1 in 10,000 live female births and is the second most common cause of severe mental retardation in females, after Down syndrome. Currently, there is no cure or effective therapy for RTT. Approved treatment regimens are presently limited to supportive management of specific physical and mental disabilities. In this issue, Krishnan and colleagues reveal that the protein tyrosine phosphatase PTP1B is upregulated in patients with RTT and in murine models and provide strong evidence that targeting PTP1B has potential as a viable therapeutic strategy for the treatment of RTT.
\end{abstract}

Methyl-CpG-binding protein 2: linking epigenetics to neuronal function

Over $95 \%$ of classic Rett syndrome (RTT) cases are linked to mutations in a single gene that encodes methyl-CpG-binding protein 2 (MECP2) $(1,2)$, one of six mammalian methyl-CpG-binding proteins identified so far. These proteins specifically bind DNA methylated at the $5^{\prime}$ position of cytosine within cytosine-guanine $(\mathrm{CpG})$ dinucleotide sequences, a process that constitutes an important gene-silencing mechanism in eukaryotes and is essential for viability (3). MECP2 dysfunction results in various neuropsychiatric disorders that include mild mental retardation, learning disabilities, autism spectrum disorders, and complex forms of severe mental retardation $(1,2)$. In RTT, numerous, mostly spontaneous mutations have been identified in $M E C P 2$ that result in a loss of MECP2 function. Several Mecp2-KO mouse models have been generated to investigate RTT pathology and to potentially identify and test new therapies (2). MECP2-deficient mice manifest many of the predominant characteristics of human RTT. The traditionally held belief is that the neuro- logical and psychiatric features caused by RTT-associated failures that occur during brain development are irreversible; however, studies in MECP2-deficient mice suggest that normal brain function can be restored by reinstating MECP2 (4). These results are promising, because they suggest that it may be possible to improve neuronal function in patients with RTT. However, therapeutic approaches aimed at altering MECP2 levels in patients will likely require a more complex strategy, as both loss of MECP2 function and elevated MECP2 levels have deleterious neurological effects. Thus, current basic and clinical research has focused on identification of critical factors downstream of MECP2 that have altered expression or function and may directly cause neuronal defects in RTT. Candidate molecules include brainderived neurotrophic factor (BDNF) and IGF-1, both of which are currently the focus of many investigations (5-8). Approaches that elevate BDNF expression and those using BDNF mimetics have shown promising results in RTT animal models (9-12), while phase I clinical trials of recombinant IGF-1 treatment in patients with RTT have produced encouraging outcomes (13).

Related Article: p. 3163

Conflict of interest: The author has declared that no conflict of interest exists.

Reference information: J Clin Invest. 2015;125(8):2931-2934. doi:10.1172/JCI83192.

PTP1B: linking MECP2 to insulin signaling

Several lines of evidence suggest that RTT is associated with metabolic disorders. First, lipid metabolism is disturbed in both RTT mouse models, in which cholesterol levels are elevated in the brain (14), and in RTT patients, in whom plasma levels of cholesterol regulatory proteins are altered (15). Second, various studies found typical signs of insulin and leptin resistance as well as obesity in RTT animal models (16, 17). Moreover, insulin resistance has also been reported in patients with RTT (18). In this issue, Krishnan and colleagues investigated the metabolic processes associated with RTT (19). The authors examined glucose metabolism and insulin signaling in male $M e c p 2^{-/ y}$ and female $M e c p 2^{-/+}$mice and found that compared with WT littermates, MECP2-deficient mice had markedly higher blood glucose levels, suggesting both glucose and insulin intolerance. Moreover, insulin-induced tyrosine phosphorylation of both the insulin receptor and insulin receptor substrate-1 (IRS1) were attenuated in $M e c p 2^{-/ y}$ and Mecp $2^{-/+}$mice, despite the increased levels of insulin in these animals compared with levels in controls. Finally, insulin-induced phosphorylation of key signaling molecules downstream of the insulin receptor, including AKT, FOXO, and GSK3 $\beta$, was attenuated in Mecp2-mutant mice. Together, these data demonstrate that typical features of insulin resistance are present in the RTT animal model. Krishnan et al. specifically tested the effects of Mecp2 loss on gene expression in the mouse forebrain to identify candidate effectors that control insulin signaling and glucose metabolism. Ptpn1, which encodes the protein tyrosine phosphatase PTP1B, was among the four genes that were substantially upregulated in MEPC2-deficient mice compared with WT animals. PTP1B is a major metabolic regulator that inhibits insulin signaling by directly dephosphorylating the insulin receptor and IRS1 (20) and reduces leptin signaling by dephosphorylating the leptin receptor-associated tyrosine kinase JAK2 $(21,22)$. Krishnan 

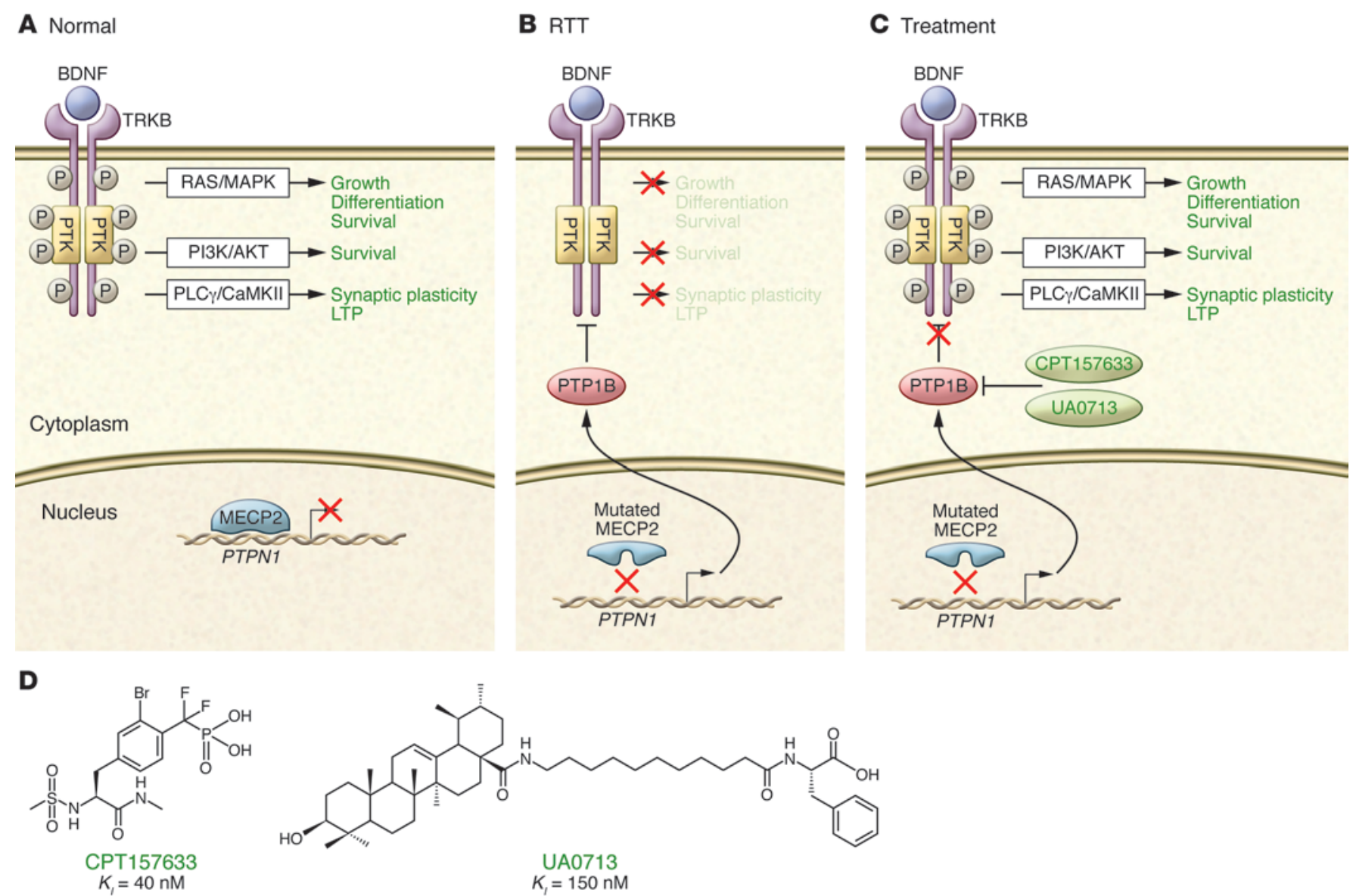

Figure 1. Reduced BDNF signaling in RTT is due to increased expression of PTP1B. (A) Binding of BDNF to its cognate receptor TRKB induces ligand-receptor dimerization and autophosphorylation of tyrosine residues (Y706 and Y707) within the intracellular protein tyrosine kinase (PTK) domain, resulting in kinase activation. Subsequently, additional tyrosine residues within the juxtamembrane domain and C-terminus of TRKB are phosphorylated and provide docking sites for adapter and signaling molecules. These initiate signaling via several pathways, including RAS/MAPK, PI3K/AKT, and PLC $\gamma / C a M K I I$, that ultimately lead to survival, growth, differentiation, synaptic plasticity, and long-term potentiation (LTP). In this issue, Krishnan and colleagues (19) demonstrated that the protein tyrosine phosphatase PTP1B directly targets the tyrosine residues within the TRKB kinase activation loop and therefore is a critical negative regulator of BDNF signaling. The transcriptional regulator MECP2 directly binds to the promoter region of PTPN1, which encodes PTP1B, and suppresses its transcription. (B) In RTT, MECP2 function is impaired due to mutations in the MECP2 gene. Consistently, PTP1B levels were upregulated in RTT patients and mouse models of RTT. (C) Specific inhibition of PTP1B activity with two small-molecule drugs (CPT157633 and UA0713) improved symptoms and survival in RTT animals, providing proof of principle for PTP1B inhibition as a potential therapeutic strategy in RTT. (D) Chemical structure of the PTP1B inhibitors CPT157633 and UA0713.

and colleagues demonstrated that MECP2 directly binds to the PTPN1 promoter and suppresses transcription. In contrast, an RTT-associated MECP2 mutant (MECP2R168X) did not suppress transcription of PTPN1. Together, these data indicate that PTPN1 is a direct target of MECP2 and that loss of MECP2 function in RTT elevates PTP1B levels and thereby impairs insulin signaling and glucose metabolism.

\section{PTP1B inhibitors: improving symptoms and survival in RTT animals}

Krishnan and colleagues (19) evaluated two previously characterized small-molecule inhibitors of PTP1B, the difluoro- methylphosphonic acid CPT157633 (23) and the ursolic acid derivative UA0713 (24), in RTT mouse models to evaluate PTP1B inhibition as a potential therapeutic strategy for RTT (Figure 1). PTP1B has been investigated as a drug target for type 2 diabetes for more than 15 years (25), and there are multiple inhibitors available. The choice of Krishnan et al. to test the PTP1B inhibitor CPT157633 seems well reasoned, given the excellent potency and relative selectivity for PTP1B demonstrated for this compound. Moreover, NMR chemical shift perturbation and x-ray crystallography experiments both confirmed that CPT157633 binds the PTP1B active site, further vali- dating CPT157633 as a potent, competitive, and reversible inhibitor. Moreover, in light of the common difficulties related to in vivo bioavailability and specificity of active-site tyrosine phosphatase inhibitors $(26,27)$, the commitment of Krishnan and colleagues to add a second compound with a presumably different mode of PTP1B inhibition is laudable. However, the choice of UA0713, a rather weakly characterized compound in terms of both PTP1B-specific binding and mode of inhibition, is somewhat less convincing, given that other options are available, including an allosteric PTP1B inhibitor with proven in vivo efficacy and mode of action previously studied 
by the same research group (28). Regardless, the data obtained from the inhibitor experiments are quite compelling. Compared with vehicle-treated controls, male Mecp $^{-/ y}$ mice given CPT157633 (5 $\mathrm{mg} / \mathrm{kg}$ daily) or UA0713 (5 mg/kg every other day) exhibited a marked reduction in glucose intolerance, an increase in body weight, and a decrease in circulating levels of insulin and cholesterol just two weeks after initiating treatment. Moreover, PTP1B inhibition improved survival of $M e c p 2^{-/ y}$ mice by increasing lifespan approximately two-fold. CPT157633 treatment yielded similar, beneficial results in female Mecp $^{-/+}$mice, which are a closer reflection of RTT in humans. In addition, PTP1B inhibitor-treated MECP2deficient mice were subjected to distinct behavioral tests, including paw clasping, regression of motor skills, and pup gathering, to assess the effect of treatment on phenotypic symptoms. Impressively, CPT157633 treatment reduced paw clasping and latency to gather pups in MECP2-deficient animals to levels observed for WT control mice. Motor skills, as measured by rotarod performance, were only partially restored by CPT157633 treatment; however, the modest improvements were lost once CPT157633 treatment was stopped, suggesting that the effects of PTP1B inhibition are reversible. On the molecular level, PTP1B inhibitor treatment resulted in enhanced tyrosine phosphorylation of both the insulin receptor and IRS1, as expected. Interestingly, other antidiabetic drugs, including metformin, rosiglitazone, and AICAR, improved glucose homeostasis in MECP2-deficient animals but did not extend lifespan, suggesting that PTP1B inhibition alters additional signaling events critical for the RTT phenotype.

\section{TRKB: linking PTP1B to BDNF signaling}

Consistent with earlier reports, BDNF levels were decreased by $30 \%$ to $40 \%$ in both $M e c p 2^{-/ y}$ and $M e c p 2^{-/+}$mice compared with levels in control animals. This observation suggested that weakened BDNF signaling through its cognate receptor tropomyosin-related kinase $\mathrm{B}$ (TRKB), but not loss of BDNF itself, could be a major contributing fac- tor to RTT development. Indeed, tyrosine phosphorylation (activation) of TRKB was attenuated in $M e c p 2^{-/+}$mice compared with that observed WT mice. Moreover, treatment with the PTP1B inhibitor CPT157633 enhanced tyrosine phosphorylation of TRKB in both WT and $\mathrm{Mecp}^{-\mathrm{I}^{+}}$mice. Krishnan et al. used a recombinant $\mathrm{PTP} 1 \mathrm{~B}$ substrate-trapping mutant (PTP1B-D181A), which forms a stable complex with the phosphorylated substrate, and demonstrated that PTP1B-D181A, but not WT PTP1B, binds to TRKB in mouse brain lysates (19). Further, PTP1B-D181A was added to lysates from cells overexpressing either WT TRKB or one of three TRKB isoforms in which tyrosine residues were mutated to phenylalanine to determine the exact tyrosine residue(s) targeted by PTP1B. The results of these assays demonstrated that PTP1B specifically targets the TRKB activation loop tyrosines Y706 and Y707 (Figure 1). Consistently, CPT157633 treatment enhanced tyrosine phosphorylation of $\mathrm{Y706/Y707}$ in the brains of Mecp $2^{-/+}$mice. Tyrosine phosphorylation of the closely related TRK receptors TRKA and TRKC was less affected by CPT157633 treatment. Together, these data demonstrate a previously unrecognized role for PTP1B as an inhibitor of BDNF signaling and suggest a possible mechanism by which PTP1B promotes RTT phenotypes. Interestingly, impaired signaling through TRKB has been associated with severe hyperphagia and obesity in both mice (29) and humans (30), suggesting that the role of PTP1B in obesity may go beyond its previously known effects on leptin receptor signaling.

\section{PTPs: drugging the undruggable}

Protein tyrosine phosphatases (PTPs), which comprise a family of more than 100 members in humans, are important signaling molecules that have been considered as potential drug targets in many human diseases and conditions, including cancer as well as cardiovascular, immunological, infectious, neurological, and metabolic diseases $(26,31)$. However, past efforts to develop drugs that target specific PTPs have been plagued by issues related to the bioavailability and selectivity of these compounds, as the vast majority of reported PTP inhibitors carry a phosphotyrosine-mimicking (pTyr-mimicking) group that interacts strongly with a highly conserved phosphate-binding loop in the active site. Consequently, active-site PTP inhibitors often fail in preclinical development due to off-target effects and suboptimal pharmacokinetic properties, which are caused by the charged nature of the pTyr mimic. Indeed, despite the great interest in PTP1B inhibitors as antidiabetic drugs over the past 15 years, few compounds have progressed to clinical trials, and so far none of these have advanced beyond phase II testing. Several new approaches to overcome these hurdles have been proposed (26). Notably, allosteric inhibition of PTPs has gained substantial traction recently, including allosteric inhibition of PTP1B (28). Previous highthroughput screening (HTS) efforts to find PTP inhibitors were mostly based on simple biochemical assays that used truncated PTPs and small pTyr mimics as substrates, a combination that favors the identification of hits that target the conserved active site. Novel HTS strategies, preferably those that can be applied under physiological conditions, will be necessary to identify PTP inhibitors that are not restricted in their mode of action, bind to allosteric sites, interfere with target localization, or block substrate binding distal from the active site. Given the undisputed importance of PTPs in disease development and progression, it is clear that investing in novel and innovative strategies to effectively target these molecules will have enormous benefits in the long term.

\section{Acknowledgments}

The author thanks Paul Lombroso and Nicholas Cosford for critical reading and editing of the manuscript. Work in the author's laboratory is supported by the American Heart Association (Innovative Research Grant 14IRG18980075) and the NIH (5R03 MH095532).

Address correspondence to: Lutz Tautz, NCI-Designated Cancer Center, SanfordBurnham Medical Research Institute, 10901 N. Torrey Pines Rd., La Jolla, California 92037, USA. Phone: 858.646.3100; E-mail:tautz@sanfordburnham.org. 
1. Chahrour M, Zoghbi HY. The story of Rett syndrome: from clinic to neurobiology. Neuron. 2007;56(3):422-437.

2. Weng SM, Bailey ME, Cobb SR. Rett syndrome: from bed to bench. Pediatr Neonatol. 2011;52(6):309-316.

3. Jaenisch R, Bird A. Epigenetic regulation of gene expression: how the genome integrates intrinsic and environmental signals. Nat Genet. 2003;33(suppl):245-254

4. Guy J, Gan J, Selfridge J, Cobb S, Bird A. Reversal of neurological defects in a mouse model of Rett syndrome. Science. 2007;315(5815):1143-1147.

5. Chen WG, et al. Derepression of BDNF transcription involves calcium-dependent phosphorylation of MeCP2. Science. 2003;302(5646):885-889.

6. Martinowich K, et al. DNA methylationrelated chromatin remodeling in activitydependent BDNF gene regulation. Science. 2003;302(5646):890-893.

7. Chang Q, Khare G, Dani V, Nelson S, Jaenisch R. The disease progression of Mecp2 mutant mice is affected by the level of BDNF expression. Neuron. 2006;49(3):341-348.

8. Tropea D, et al. Partial reversal of Rett Syndromelike symptoms in MeCP2 mutant mice. Proc Natl Acad Sci U S A. 2009;106(6):2029-2034.

9. Lauterborn JC, Pineda E, Chen LY, Ramirez EA, Lynch G, Gall CM. Ampakines cause sustained increases in brain-derived neurotrophic factor signaling at excitatory synapses without changes in AMPA receptor subunit expression. Neuroscience. 2009;159(1):283-295.

10. Deogracias R, et al. Fingolimod, a sphingosine-1 phosphate receptor modulator, increases BDNF levels and improves symptoms of a mouse model of Rett syndrome. Proc Natl Acad Sci U S A. 2012;109(35):14230-14235.

11. Schmid DA, et al. A TrkB small molecule partial agonist rescues TrkB phosphorylation deficits and improves respiratory function in a mouse model of Rett syndrome. J Neurosci. 2012;32(5):1803-1810.

12. Kron M, Lang M, Adams IT, Sceniak M, Longo F, Katz DM. A BDNF loop-domain mimetic acutely reverses spontaneous apneas and respiratory abnormalities during behavioral arousal in a mouse model of Rett syndrome. Dis Model Mech. 2014;7(9):1047-1055

13. Khwaja OS, et al. Safety, pharmacokinetics, and preliminary assessment of efficacy of mecasermin (recombinant human IGF-1) for the treatment of Rett syndrome. Proc Natl Acad Sci U S A. 2014;111(12):4596-4601.

14. Buchovecky CM, et al. A suppressor screen in Mecp2 mutant mice implicates cholesterol metabolism in Rett syndrome. Nat Genet. 2013;45(9):1013-1020.

15. Segatto M, et al. Cholesterol metabolism is altered in Rett syndrome: a study on plasma and primary cultured fibroblasts derived from patients. PLoS One. 2014;9(8):e104834.

16. Fyffe SL, et al. Deletion of Mecp2 in Sim1expressing neurons reveals a critical role for MeCP2 in feeding behavior, aggression, and the response to stress. Neuron 2008;59(6):947-958.

17. Pitcher MR, et al. Insulinotropic treatments exacerbate metabolic syndrome in mice lacking MeCP2 function. Hum Mol Genet. 2013;22(13):2626-2633.

18. Cooke DW, Naidu S, Plotnick L, Berkovitz GD. Abnormalities of thyroid function and glucose control in subjects with Rett syndrome. Horm Res. 1995;43(6):273-278.

19. Krishnan N, et al. PTP1B inhibition suggests a therapeutic strategy for Rett syndrome. J Clin Invest. doi:10.1172/JCI80323.

20. Elchebly M, et al. Increased insulin sensitivity and obesity resistance in mice lacking the protein tyrosine phosphatase-1B gene. Science.
1999;283(5407):1544-1548.

21. Zabolotny JM, et al. PTP1B regulates leptin signal transduction in vivo. Dev Cell. 2002;2(4):489-495.

22. Cheng A, et al. Attenuation of leptin action and regulation of obesity by protein tyrosine phosphatase 1B. Dev Cell. 2002;2(4):497-503.

23. Blaskovich MA et al., inventors; Ceptyr Inc., assignee. Protein tyrosine phosphatase inhibitors methods of use thereof. US Patent 7504389B2. March 17, 2009.

24. Zhang W, et al. Ursolic acid and its derivative inhibit protein tyrosine phosphatase $1 \mathrm{~B}$, enhancing insulin receptor phosphorylation and stimulating glucose uptake. Biochim Biophys Acta. 2006;1760(10):1505-1512.

25. Tiganis T. PTP1B and TCPTP - nonredundant phosphatases in insulin signaling and glucose homeostasis. FEBS J. 2013;280(2):445-458.

26. Tautz L, Critton D, Grotegut S. Protein tyrosine phosphatases: structure, function, and implication in human disease. Methods Mol Biol. 2013;1053:179-221.

27. He R, Zeng LF, He Y, Zhang S, Zhang ZY. Small molecule tools for functional interrogation of protein tyrosine phosphatases. FEBS J. 2013;280(2):731-750.

28. Krishnan N, et al. Targeting the disordered C terminus of PTP1B with an allosteric inhibitor. Nat Chem Biol. 2014;10(7):558-566.

29. Xu B, et al. Brain-derived neurotrophic factor regulates energy balance downstream of melanocortin-4 receptor. Nat Neurosci. 2003;6(7):736-742.

30. Yeo GS, et al. A de novo mutation affecting human TrkB associated with severe obesity and developmental delay. Nat Neurosci. 2004;7(11):1187-1189.

31. Tonks N. Protein tyrosine phosphatases: from genes, to function, to disease. Nat Rev Mol Cell Biol. 2006;7(11):833-846. 